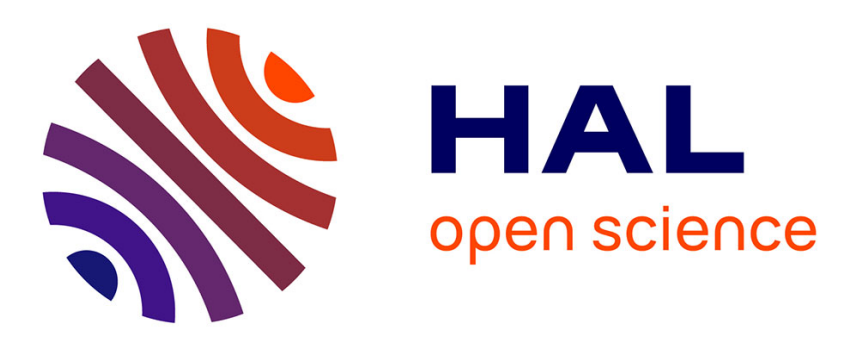

\title{
Marchandisation du travail et différenciation sociale en Sella Limba (Sierra Leone) de 1950 à nos jours.
} Augustin Pallière, Jean-Luc Paul, Hubert Cochet, Frédéric Landy

\section{To cite this version:}

Augustin Pallière, Jean-Luc Paul, Hubert Cochet, Frédéric Landy. Marchandisation du travail et différenciation sociale en Sella Limba (Sierra Leone) de 1950 à nos jours. . Journal des Africanistes, 2014, 83-2, pp.116-141. hal-01612587

\section{HAL Id: hal-01612587 \\ https://hal.science/hal-01612587}

Submitted on 13 Feb 2018

HAL is a multi-disciplinary open access archive for the deposit and dissemination of scientific research documents, whether they are published or not. The documents may come from teaching and research institutions in France or abroad, or from public or private research centers.
L'archive ouverte pluridisciplinaire HAL, est destinée au dépôt et à la diffusion de documents scientifiques de niveau recherche, publiés ou non, émanant des établissements d'enseignement et de recherche français ou étrangers, des laboratoires publics ou privés. 


\section{Marchandisation du travail et différenciation sociale en Sella Limba (Sierra Leone) de 1950 à nos jours}

Augustin Palliere, doctorant AgroParisTech, UMR Prodig / Université Paris Ouest Nanterre La Défense, UMR LAVUE

Jean-Luc Paul, Anthropologue UMR 8171 CEMAF / Université Antilles Guyane

Hubert Cochet, Professeur AgroParisTech, UFR Agriculture Comparée et Développement Agricole, UMR PRODIG

Frédéric Landy, Géographe, Université Paris Ouest Nanterre La Défense, UMR LAVUE

\section{Résumé (Français) :}

En Sierra Leone, les migrations vers les zones diamantifères et le développement de cultures à vocation commerciale ont contribué à l'intégration croissante des paysanneries à l'économie nationale et mondiale. Sur la base d'une étude d'une petite région du nord du pays, la Sella Limba, nous décrivons comment la marchandisation des rapports sociaux de production et d'échanges a impliqué le détournement des rapports sociaux «traditionnels» fondés sur l'antériorité et l'éclatement des grands groupes domestiques en unités de plus petite taille à l'existence plus précaire. Mais, marginalisées par les importations massives à bas prix de riz, ces agricultures manuelles sont restées à très faible niveau de capital. Dans ce contexte, nous montrons comment la faiblesse de la productivité du travail limite les possibilités de différenciation sociale et économique durables. Les paysans combinent des rapports sociaux autant «modernes» que «traditionnels» pour développer des stratégies d'accumulation hybrides qui relèvent parfois plutôt de la simple survie.

\section{Résumé (Anglais) :}

In Sierra Leone, the migration to the diamond fields and the development of cash crops have contributed to the increasing integration of the peasantry in the national and global economy. Based on a study of a small northern chiefdom, the Sella Limba, we describe how the commodification of social relations of production and exchange involved the perversion of "traditional" social relations based the anteriority and finally the break-up of the large domestic groups into smaller units more precarious. But marginalized by massive cheap rice 
imports, these manual agricultures remained at very low level of capital intensity. We look how low labor productivity limits the opportunities for sustainable social and economic differentiation. Farmers combine "modern" and "traditional" social relations and develop hybrid accumulation strategies sometimes coming close to mere survival.

\section{Introduction}

La « modernisation » des sociétés rurales africaines renvoie couramment, dans la littérature scientifique, aux dynamiques induites par la pénétration des rapports marchands: monétarisation de l'économie, déstructuration des structures lignagères, développement des migrations économiques, scolarisation des enfants... Bernstein (2010) dans Class Dynamics of Agrarian Change défend l'idée qu'avec la marchandisation des produits agricoles les paysans devenus «petits producteurs de marchandises » sont soumis à une différenciation en « classes ». Si l'on constate en effet ce processus dans certaines régions où l'agriculture marchande s'est développée précocement, dans beaucoup d'autres régions, en Afrique notamment, aucune accumulation n'a accompagné la «modernisation ». Mises en concurrence sur le marché mondial avec des agricultures beaucoup plus performantes (Mazoyer \& Roudart 1997: 445-481), ces paysanneries ont été marginalisées \& ont constituées une réserve de main d'œuvre bon marché pour le secteur capitaliste (Meillassoux $1975: 139-205)$.

En Sierra Leone, l'intégration de la paysannerie à l'économie de marché, bien qu'aujourd'hui structurelle et irréversible, est encore fort incomplète, notamment dans les zones enclavées comme c'est le cas de notre terrain de recherche, la Sella Limba, au Nord du pays. Ici, à partir des années 1950, l'exploitation diamantifère entraîne des migrations de travail et ouvre la voie à la commercialisation des produits vivriers. Plus tard, des cultures purement commerciales (tabac puis piment) apparaissent. Ces dernières années enfin, l'orpaillage artisanal offre de nouvelles opportunités de travail salarié. Cette intégration ne s'accompagne cependant d'aucun bouleversement des techniques de production agricole qui restent exclusivement manuelles et dont la productivité se dégrade. Bien que la marchandisation des relations sociales s'est accompagnée de l'éclatement des groupes domestiques $^{1}$ en unités de plus faible taille et à l'existence souvent précaire, l'organisation

1 Défini au sens de l'anthropologie économique (Meillassoux 1975). En Sella Limba, le groupe domestique est longtemps resté le lieu où se décidait et se déroulait l'essentiel de la circulation du travail et de son produit 
sociale de la paysannerie continue de porter l'empreinte forte des anciennes solidarités.

Malgré le maintien des paysans sella limba dans une grande pauvreté matérielle et l'absence d'accumulation de capital au sens classique du terme, la marchandisation est-elle, comme le prédit Bernstein, synonyme de différenciation économiques et sociales durables au sein de ces paysanneries ? C'est la question à laquelle cet article tente de répondre.

L'étude historique détaillée, sur 3 à 4 générations de producteurs, des rapports sociaux qui règlent la circulation et le partage de la force de travail permettra dans un premier temps de comprendre la situation actuelle des groupes domestiques, caractérisées par l'instabilité de leurs contours et l'évolution permanente de leur fonctionnement. On verra ensuite que les institutions qui encadrent aujourd'hui l'essentiel des échanges de travail entre les groupes domestiques et les individus offre la possibilité de décomposer la force de travail mais freine le développement de sa marchandisation totale. Sur cette base, on analysera les formes que prend aujourd'hui, malgré la faiblesse des niveaux de productivité, la différenciation des unités de production / consommation.

L'essentiel des sources de l'analyse historique est constitué par les témoignages de paysans sella limba, des deux sexes et de tout âge, réalisés dans le cadre d'un doctorat entre mars 2007 et décembre 2011. A travers la reconstitution des trajectoires individuelles et des groupes domestiques, ces entretiens avaient pour objet l'analyse de l'évolution des pratiques paysannes et des rapports de production et d'échange. La dernière partie de l'article repose sur l'exploitation de 58 études de cas conduites en Sella Limba entre 2010 et $2011^{2}$.

\section{Le groupe domestique durant la première moitié du XXe siècle : circulation interne du travail et des subsistances}

Avec plus de 30 hab / km² (McCulloch 1964 : 49), la Sella Limba était déjà, en 1930, une zone densément peuplée pour la région. Les recrûs arborés, associant lianes et arbres à croissance rapide dans une formation végétale dense (thakay), dominaient alors un paysage de collines. On les exploitait en abattis-brûlis : défrichés à la hache et au sabre d'abattis, ils étaient laissés à sécher puis brûlés pendant la période la plus chaude de l'année. En début de saison des pluies, le semis du riz, associé à un complexe cultural très riche, était accompagné

2 La constitution de notre échantillon a reposé sur un zonage agro-écologique (Palliere, 2013) et sur les résultats de notre analyse historique. 
d'un grattage superficiel à l'aide d'une houe à soie au fer étroit (kusala). L'entretien des parcelles mobilisait les femmes (désherbage) comme les hommes (construction de barrières) avant les récoltes qui s'étalaient de la fin de la saison des pluies au début de la saison sèche. En seconde année, arachides et fonio étaient cultivés sur une partie de la parcelle. Ensuite, la reproduction de la fertilité était assurée par un recrû arboré d'une période de 7 à 10 ans.

Le groupe domestique était constitué du «cercle des maisons» (kuru kuru). Chaque hameau (meti) comprenait deux à trois kuru kuru. A la tête de chacun d'entre eux se trouvait un homme âgé que nos témoins désignent comme le « père » (handa), « l'aîné ${ }^{3}$ » (mayon ma, le premier) ou le «chef» $(g a b k u)$. Chaque année, il désignait l'emplacement du «grand essart» (tembuy). Tous les hommes et toutes les femmes du kuru kuru cultivaient cette parcelle et la récolte de riz paddy (paga) était placée dans des coffres en bois, sous le contrôle strict de l'aîné, responsable de la conservation des semences et de la distribution du paddy pour la consommation courante du kuru kuru. Le paddy était confié aux femmes les plus âgées disposant chacune de son propre foyer (ou marmite, somba). Elles supervisaient la préparation quotidienne de la nourriture et répartissaient le riz cuit (sisa) entre les individus sous leur responsabilité.

Les récits témoignent de tensions qui traversaient le kuru kuru, tensions d'autant plus vives qu'augmentait sa taille, et qui se résolvaient par sa segmentation. Fonder un nouveau hameau aux marges du territoire cultivé, ou un nouveau kuru kuru au sein du hameau initial, était une option réservée aux hommes mariés, disposant de leurs propres outils et semences et capables d'entraîner un nombre conséquent de dépendants. Une solution provisoire consistait à confier à un groupe d'actifs la culture d'un petit essart (hutolo), toujours contigu au tembuy. Le produit du petit essart, comme celui des parcelles de fonio et d'arachide de seconde année, ne rejoignait pas le coffre de l'aîné mais restait sous le contrôle de la femme gestionnaire de la « marmite » du groupe concerné. Mais grands et petits essarts étaient toujours jointifs et un unique brûlis était réalisé au moment jugé opportun par l'aîné. La fenêtre calendaire pour la défriche des petits essarts des cadets, et par conséquent la taille de ceux-ci, restaient donc contraintes par le calendrier agricole contrôlé par l'aîné.

Le contrôle de l'aîné sur les cadets se traduisait donc, à l'échelle du cycle agricole, par une organisation stricte de l'assolement et des calendriers de travail. Mais ce contrôle reposait

3Dans la suite du texte nous utiliserons ce terme qui fait référence à une certaine institutionnalisation du rapport d'antériorité vis à vis des « cadets » (Paul 2003 : 309) 
surtout, à l'échelle des cycles viagers, sur celui de la circulation des épouses entre les hameaux ${ }^{4}$. Ce sont l'aîné et les aînées, à la tête respectivement du kuru kuru et des «marmites », qui procuraient à leurs fils des épouses. Elles provenaient d'une aire matrimoniale ${ }^{5}$ constituée de plusieurs hameaux voisins. Tous les témoins insistent sur l'âge tardif auquel les jeunes hommes se mariaient alors. Au contraire, les jeunes filles partaient très jeunes vivre chez leurs affins (komone), dans le kuru kuru de leur futur époux. Elles y travaillaient de longues années sous l'autorité de leur belle-mère avant de disposer à leur tour de leur propre «marmite ». La « patience » dont les cadet(te)s faisaient preuve était alors la manifestation du « respect » témoigné envers leurs aîné(e)s.

Durant les premières décennies du protectorat britannique, décrété en 1896, la Sella Limba resta isolée par rapport aux régions proches des comptoirs commerciaux de l'embouchure des rivières du nord ou des stations chemin de fer achevé en 1907 (Riddell 1970 ; Deveneaux 1973 ; Sibanda 1979; Alie 1990). Jusqu'au début des années 1950, le système social traditionnel semble avoir échappé aux involutions radicales subies dans d'autres régions plus exposées aux processus de modernisation.

Les opportunités économiques permettant aux cadets d'échapper au contrôle des aînés, opportunités qui se multiplieront durant les dernières années du protectorat, étaient alors très limitées. Les sella limbas ne vivaient pas pour autant en autarcie : la vente des noix de palmistes, notamment, récoltées tout au long de l'année, procurait un revenu monétaire. Mais le comptoir commercial le plus proche se trouvait à une centaine de kilomètres qu'il fallait parcourir à pied durant le creux du calendrier de travail qui suivait la récolte. La part du produit agricole consacrée à la vente restait donc faible et, bien que le voyage fut entrepris par les cadets, les recettes monétaires restaient sous contrôle de l'aîné. Elles lui permettaient d'acquitter l'impôt de case du protectorat (hut tax).

Cette amorce de marchandisation et de monétarisation de l'activité agricole a-t-elle constitué le ferment de l'émancipation future des cadets ? L'intégration de la paysannerie sella limba à l'économie nationale restait en tous cas trop faible pour que ces potentialités s'expriment: le groupe domestique produisait la quasi-totalité de sa subsistance, la plus grande part du travail des productifs était consacrée à la production vivrière et seule une part marginale du produit agricole faisait l'objet d'échanges marchands. Ainsi, le grand essart de

4 La société sella limba est patrilinéaire et virilocale.

5 Meillassoux (1975: 73). 
l'aîné absorbait la quasi-totalité du travail des individus productifs du kuru kuru, tandis que la quasi-totalité du produit agricole circulait en sens inverse, du coffre de l'aîné aux marmites des femmes aînées.

Dans cette économie très faiblement intégrée au marché, la production agricole n'avait de valeur qu'en tant que subsistance susceptible de se convertir en énergie humaine ${ }^{6}$ à court ou moyen terme (entretien des productifs et production des producteurs), dans le cadre contraignant de la reproduction du système social. Les déséquilibres démographiques structurels entre productifs et improductifs, comme il ne manque pas d'en advenir au sein de groupes numériquement faibles, se résolvaient par la mise en circulation des enfants préproductifs. Cette circulation assurait l'équilibre entre la capacité productive du groupe et le nombre de bouches à nourrir. Elle se réalisait d'abord au sein même du kuru kuru, entre les marmites des grand-mères paternelles (nanda) qui avaient la responsabilité de nourrir les rejetons de leurs belles-filles. Il était aussi courant pour qu'une grand-mère maternelle élève le rejeton de sa fille mariée. L'enfant quittait alors le kuru kuru de son père pour rejoindre celui de son oncle utérin (sisa), parfois définitivement. Aujourd'hui encore se côtoient dans les hameaux sella limba des lignages issus en ligne patrilinéaire des fondateurs supposés (lignages lasiri) et des lignages, plus ou moins anciens, identifiés comme les sisa perpétuels des premiers, originaires d'un des villages de l'aire matrimoniale (lignages thahine). Jusqu'à cette époque, la circulation du travail n'avait donc pas encore revêtu sa forme moderne de « force de travail », c'est-à-dire de quantités discrètes de travail en partie autonomisées du reste de la vie sociale. C'est par le mariage virilocal et par l'adoption ou le fosterage que des individus, dans leur plénitude sociale, étaient intégrés au groupe qui allait bénéficier de leurs capacités productives présentes ou à venir.

\section{Du grand essart de riz aux petites parcelles de piment :} marchandisation de la force de travail et du produit agricole en Sella Limba depuis 1950

Dans les années 1950, le secteur diamantifère connut un essor spectaculaire à l'est du pays. L'extraction manuelle des diamants alluviaux, longtemps interdite, fut autorisée en 1956 (Zack-Williams 1982). Industrie à très faible niveau de capital, les mines nécessitaient une importante main d'œuvre. Pour les jeunes hommes sella limba, c'était une opportunité

6 Meillassoux $(1975: 83)$ 
nouvelle. Leur travail acquit une valeur d'échange dont l'actualisation impliquait de quitter hameau et groupe domestique. Choix difficile car il était synonyme de rupture d'avec le lignage. Les aînés ne pouvaient en effet accepter d'être privés de ces productifs qu'ils avaient eux-mêmes «produits » et dont la présence au sein du kuru kuru était la condition sine qua none à son bon fonctionnement - comme elle l'était d'ailleurs à la reproduction de l'ensemble du système social.

De leur côté, les aînés participèrent à la déstructuration des kuru kuru. Le développement du secteur minier créa une forte demande en vivrier pour nourrir les mineurs (Levi 1975 ; Richards 1996: 51). Rapidement, des commerçants s'installèrent en Sella Limba à la recherche de riz à revendre dans les districts diamantifères. Ils proposaient en échange aux paysans de nouveaux produits manufacturés. C'est alors, par exemple, que la tôle ondulée s'imposa comme l'attribut indispensable de la maison d'un chef de famille respectable. Ainsi, la production vivrière acquit elle aussi une valeur d'échange. La double circulation interne aux kuru kuru du produit et du travail fut en partie détournée vers le marché. Les témoignages sont nombreux qui attestent de la multiplication des tensions entre les générations engendrées par ces évolutions. On évoque les frères cadets partis «sans même dire au revoir » ou encore les menaces et les pratiques de sortilèges des aînés à l'encontre des cadets. Jusqu'aux années 1990, c'est environ la moitié de chaque fratrie qui quittera les hameaux, le plus souvent pour les mines de diamant. L'énergie humaine investie dans la production des producteurs fut donc en grande partie détournée au profit du secteur minier. Il faut souligner que les salaires journaliers des mineurs n'excédaient guère le minimum nécessaire à la simple reproduction de la force de travail, au jour le jour, ce qui suggère que le départ vers les mines correspondait pour les cadets autant à une aspiration à l'émancipation qu'au désir d'amélioration de leurs conditions matérielles de vie.

A cette même époque, les paysans sella limba adoptèrent les techniques de la riziculture inondée de bas-fonds. Elles permettaient à la fois de mieux répondre à l'opportunité offerte par l'émergence du marché vivrier et de retenir une partie des jeunes au village. Un nouveau compromis fut trouvé au sein des groupes domestiques : en échange du travail fourni sur le tembuy des versants, les aînés offrirent aux cadets la possibilité de cultiver à leur compte une parcelle de riz inondée dans les bas-fonds, souvent par groupe de frères utérins : après les semailles dans les champs pluviaux, les jeunes travailleurs descendaient labourer les basfonds, utilisant une houe à soie au fer large d'un nouveau type (kubada), et repiquer le riz. 
Dans les bas-fonds, pour suivre les mouvements de la lame d'eau, il fallait effectuer rapidement ces opérations. L'organisation des jeunes en groupes saisonniers (kune), qui travaillaient alternativement dans les champs de bas-fonds des uns et des autres, répondit en partie à cette nouvelle contrainte. La récolte du riz inondé avait lieu après celle du tembuy. Une partie du riz de ces bas-fonds pouvait être destinée à la vente pour l'achat, par exemple, de vêtements de prestige, une autre partie permettait aux aînées maîtresses des marmites de disposer de leur propre réserve indépendante du grenier de l'aîné. Cet arrangement n'était pas entièrement nouveau. Cependant, la déconnexion spatiale entre le grand essart de l'aîné (tembuy) et les petites parcelle des cadets (hutolo) comme le développement de nouvelles techniques de culture singularisaient le travail des cadets et leur offrait la possibilité d'une organisation plus autonome. C'est ainsi que la commercialisation du riz et l'introduction concomitante du riz de bas-fonds correspondirent à un mouvement significatif d'émancipation des cadets vis-à-vis des aînés et d'autonomisation relative des différentes marmites vis-à-vis du kuru kuru?

La riziculture inondée représentait aussi un mode d'exploitation du milieu nouveau. Il ne reposait plus sur le recrû spontané entre deux cycles de culture mais sur l'aménagement de la parcelle cultivé : défrichage, dessouchage, élargissement, drainage, planage... Les paysans réalisèrent dès cette époque un investissement pluriannuel et durable en travail et, en conséquence, les actifs concernés du kuru kuru, généralement une phratrie, s'approprièrent de facto la parcelle aménagée. Cette appropriation amorça le processus d'éclatement ${ }^{8} \mathrm{du}$ kuru kuru: disposant désormais de leurs propres stocks de semences et parcelles, les groupes de frères utérins saisirent plus systématiquement l'occasion du décès de l'aîné pour refuser la succession collatérale. Refusant de se placer sous l'autorité d'un frère cadet du défunt, ils constituaient de nouveaux groupes autonomes. On aboutit donc à moyen terme à la multiplication d'unités de plus petite taille dont les caractéristiques sociodémographiques s'opposaient désormais au fonctionnement quasi-autarcique des anciens kuru kuru.

Cependant, le développement du secteur vivrier marchand va être précocement sacrifié par les importations massives de riz asiatique destinées à contenir les salaires des mineurs (Richards 1996 : 51). C'est dans ce contexte qu'une filiale locale de la British and American

7 Delarue (2007 : 200-201) décrit le même phénomène en Guinée Forestière, à la même époque.

8 On différencie ici la segmentation, processus structurel au système social « traditionnel » qui constitue le moyen de résolution de ses contradictions internes, de l'éclatement, tendance historique décrite ici et observée par de nombreux auteurs de la région (Binder 1989 ; Leach 1994 ; Delarue 2007). 
Tobacco obtint du gouvernement le monopole régional de la production et de la commercialisation du tabac. Elle mit en place un modèle de culture intégrée : en échange de l'avance des frais de campagne, notamment des engrais de synthèse, les paysans s'engageaient à suivre un itinéraire technique précis et à vendre l'intégralité de leur tabac à la compagnie. A partir de 1975, la filière intégra un grand nombre de paysans, avec de lourdes conséquences sur le mode de mise en valeur du milieu : une clause du contrat imposait le dessouchage des recrûs arborés, bouleversant les modes de reproduction de la fertilité. Désormais, celle-ci ne reposait plus sur le recrû spontané mais sur l'apport d'engrais. De plus, l'itinéraire technique du tabac entrait en concurrence avec celui du riz pluvial pour la mobilisation de la main d'œuvre. La compagnie avançait également aux tabaculteurs du riz importé, payable à la récolte : les sacs de riz asiatique prirent à cette époque une place dans l'économie locale qu'ils ne céderont plus. La période du tabac (1975-1996) est donc caractérisée par l'accentuation de l'intégration économique de la paysannerie sella limba, par une monétarisation rapide de son économie.

Le développement d'une culture à vocation uniquement commerciale établit un rapport presque direct, au sein des groupes domestiques, entre la journée de travail et sa valeur monétaire. Les échanges marchands de force de travail entre paysans - journées de travail contre argent ou contre riz - émergèrent alors. Les jeunes d'alors racontent encore aujourd'hui l'agacement qu'ils ressentaient à travailler pour que l'aîné s'enrichisse à leurs dépens. Inversement, s'offrit aux ainés, pour la première fois, la possibilité de mobiliser ponctuellement, aux moments les plus opportuns, des travailleurs dont ils n'avaient pas à assurer l'entretien l'année durant. Cette dynamique accéléra encore l'éclatement des kuru kuru et la constitution d'unités de production de petite taille.

La fin des années 1990 est marquée, avec la propagation de la guerre civile dans la région étudiée $^{9}$, par la disparition de la filière tabac et le ralentissement des activités commerciales. Le mouvement d'intégration au marché de l'économie agricole sella limba marque le pas voire recule. Aujourd'hui, le piment à destination des marchés urbains a remplacé le tabac comme culture commerciale principale et les groupes domestiques continuent de se procurer sur le marché près de la moitié des céréales qu'ils consomment. Les échanges marchands de

9 La guerre civile démarre en mars 1991, mais n'affecte directement la Sella Limba qu'à partir de 1996 et surtout d'octobre 1998, quand les rebelles du RUF installent leurs quartiers dans le bourg de Kamakwie, jusqu'à la fin officiel du conflit 3 ans plus tard. Le RUF a peu recruté parmi les jeunes ruraux de Sella Limba et la chefferie n'a pas connu d'afflux massif de réfugiés. 
force de travail - sous la forme d'achat et de vente de journées de travail entre les paysans continuent de se développer.

L'augmentation de la densité de population, associée à l'arrachage obligatoire des recrûs arborés pour la tabaculture, a entraîné la disparition de l'abattis-brûlis comme mode de mise en valeur du milieu et de reproduction de la fertilité (Palliere 2013). Pour faire face à la dégradation brutale des conditions agro-écologiques, les paysans généralisent alors la technique du billonnage qui correspond à un labour stricto sensu : par l'enfouissement de la biomasse végétale, elle limite la pression des adventices sur la culture et concentre dans l'horizon superficiel du sol la matière organique en décomposition. Le billonnage régulier des parcelles - tous les ans dans les bas-fonds avant le repiquage du riz, et tous les 4 ans sur les terres pluviales, avant le repiquage du piment - est aujourd'hui la base du mode d'exploitation du milieu en Sella Limba. Malgré ce labour de début de cycle, des désherbages plus fréquents et plus lourds sont nécessaires à l'entretien des parcelles pendant la saison des pluies. Ces nouvelles pratiques témoignent de l'intensification par le travail des systèmes de culture. Malgré les innovations de ce dernier demi-siècle, dans le domaine des variétés, des outils (qui restent cependant manuels), des rotations et associations, la productivité brute journalière du travail agricole ${ }^{10}$ des paysans a nettement décliné. Nous l'avons évalué pour aujourd'hui de 2,7 et $3,6 €^{11}{ }^{12}$. On verra plus loin que le niveau de la production n'excède guère les besoins nutritionnels nécessaires à la reproduction du groupe domestique.

Depuis 2007, avec l'évolution des prix relatifs, l'exploitation artisanale à petite échelle de l'or alluvial, en Sella Limba même, s'est fortement développée. Les jeunes sont nombreux à vendre leurs bras aux petits patrons de l'orpaillage. Mais les salaires journaliers sont maintenus entre 1,8 et 2,7€ par jour de travail, soit moins que la productivité agricole brute journalière ${ }^{13}$. Ainsi, si cette activité peut venir compléter, en saison sèche notamment, la

10 Rapport entre la quantité de produit agricole obtenu et le nombre de jours de travail investis dans la culture. 11 Pour faciliter la compréhension du texte, les sommes sont indiquées en euros, à la décimale près. En 2011, durant la période de recherche de terrain, 1 euro équivalait en moyenne à 5500 leones.

12 Ces données, qui datent de 2011, sont le résultat de l'analyse des pratiques agricoles à l'échelle des systèmes de culture basée la décomposition du paysage en facettes agro-écologiques et d'un zonage de la région étudiée (sur ces concepts : Sébillotte 1976; Cochet 2011a et 2012). Cette analyse dépasse le cadre de ce document, notons cependant que la quantification des niveaux de la productivité journalière à l'échelle des systèmes de culture intègre aussi bien les parts vendue qu'autoconsommée du produit agricole.

13 Ces salaires journaliers correspondaient en 2011 à environ 2,8 à 4,3 kg de riz décortiqué acheté sur le marché au prix moyen. Il est frappant qu'à la veille de la seconde guerre mondiale, les manœuvres sans qualification se battaient pour obtenir une rémunération journalière minimum de l'ordre de 1 shilling, correspondant à environ 5 $\mathrm{kg}$ de riz au tarif de l'époque (circulaire «Rates of unskilled labour $» \mathrm{n}^{\circ} 18 / \mathrm{D} / 9 / 31$ du 26 mai 1938). En Sierra Leone, la grande majorité des travailleurs des villes comme des campagnes en sont toujours à lutter pour leur survie. 
faiblesse des revenus agricoles toujours soumis aux aléas climatiques et à la variation des prix sur le marché, les paysans ont été peu nombreux jusqu'ici à abandonner leurs activités agricoles.

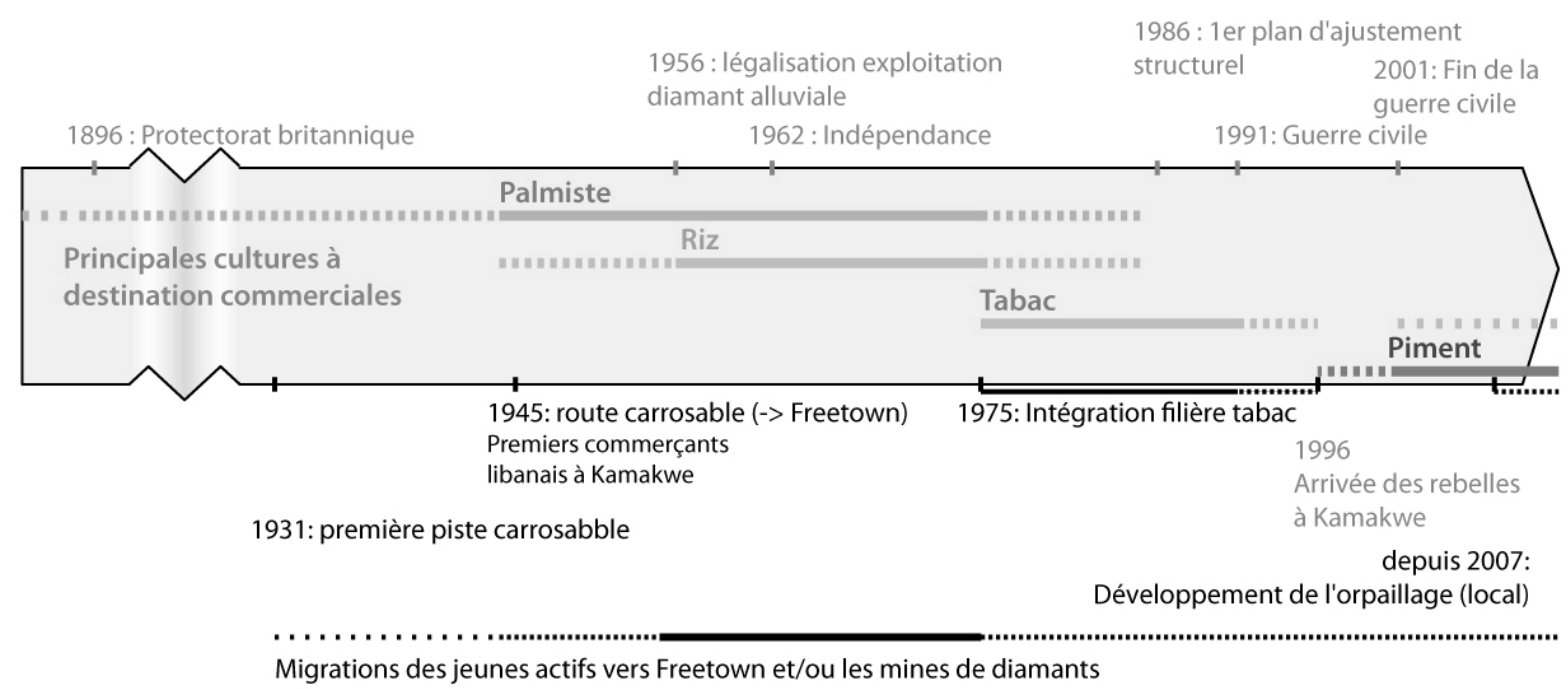

Figure 1 : Chronologie de l'intégration économique de la Sella Limba au marché national et mondial

\section{La kune: l'institutionnalisation d'une marchandisation incomplète des rapports sociaux.}

L'histoire récente de la paysannerie sella limba est donc marquée par son intégration croissante, dans des conditions variables mais tendanciellement toujours plus défavorables, à l'économie nationale et mondiale, et en conséquence par la marchandisation des rapports sociaux, c'est-à-dire par la circulation marchande du travail et du produit de ce travail. Cette dynamique - à la fois agro-écologique, sociale et économique - s'est traduite par l'éclatement du kuru kuru en groupes plus petits et plus précaires. Aujourd'hui, au sein des hameaux, ces groupes échangent des journées de travail et la grande majorité de ces échanges sont institutionnalisés en groupes de travail saisonniers, les kune. L'analyse du fonctionnement de cette institution et des usages qu'en font les paysans montre le caractère incomplet et hybride de la marchandisation des rapports sociaux.

«You work for me, I work for you » répètent à l'envi les paysans pour souligner la réciprocité et la solidarité qui règnent au sein des kune. Ils justifient cette organisation par l'effet d'entraînement et de compétition qui pousserait chacun à abattre plus de travail que s'il 
était seul. Ce type de groupe est très commun en Afrique de l'Ouest, et la littérature se fait l'écho des mêmes justifications. Ces descriptions normatives masquent le fonctionnement réel des kune dont l'analyse révèle des stratégies de captation de travail ${ }^{14}$. Tandis que certaines de ces stratégies tendent à prolonger les anciens rapports sociaux, d'autres soulignent clairement la dynamique de leur marchandisation.

\subsection{Le fonctionnement des kune : échanges, achats-ventes et dons}

La contribution de chaque membre de la kune est soumise à une comptabilité rigoureuse. Pour une kune de 20 membres, par exemple, chaque membre dispose de 5 tours. Chaque travailleur accomplit en une journée un certain nombre de portions (bara) et le chef choisi par la kune est responsable du partage équitable de ces portions. Les retards et le travail bâclé sont punis. Un adolescent effectue des demi-bara et ne bénéficie donc de la kune qu'un tour sur deux. Le souci de décomposer le travail de chaque actif de la kune trahit la manipulation des échanges entre groupes domestiques que permet cette organisation du travail, et facilite leur marchandisation. Il est en effet possible de vendre son tour de kune en échange de riz ou d'argent. Si certains paysans s'y résolvent, c'est toujours par défaut de capital circulant. Telle parcelle destinée au riz pluvial est prête à être ensemencée mais il manque les semences. On échange alors avec un paysan disposant d'un surplus de semences un tour de kune de 20 membres contre 20 pans $^{15}$ de semences ou 18,2 €. Or, le coût d'un homme-jour au sein d'une kune est inférieur à la productivité journalière moyenne agricole, ce qui justifie l'investissement des acheteurs. Néanmoins, ces derniers doivent avoir assez de capital circulant pour acheter 20 journées de travail d'un coup : la kune ne se divise pas. Des tours de kune sont également vendus pour se procurer de la nourriture pendant la soudure. Au cœur de la saison des pluies, alors que chacun se consacre à lutter contre l'enherbement de ses parcelles, la vente d'une kune de 20 membres peut permettre à une femme de nourrir 5 personnes en riz importé pendant une dizaine de jours.

Outre cette forme d'achat / vente directe, il existe une autre forme d'inégalité de l'échange de journées de travail, plus courante et plus discrète. Quand viennent leur tour d'accueillir la kune, les paysans doivent fournir un copieux repas de mi-journée. Certains membres ne disposent pas, au moment voulu, des ressources nécessaires à la préparation de ce repas. Dans ce cas, le groupe travaillera uniquement la matinée, abattant la moitié du travail normal.

14 Voir Pillot (1993 : 195-248) comme exemple d'une analyse détaillée de ce type de stratégie dans le cas d'Haïti.

15 Le pan (une bassine de plastique) de riz paddy en Sella Limba pesait en 2011, environ 2,5 $\mathrm{kg}$ de paddy. 
Ainsi, par manque structurel ou conjoncturel de trésorerie ou de réserves, certains paysans échangent des journées entières de travail contre des demi-journées.

Le don de kune, enfin, est une autre forme d'échange non équivalent. Or, ce que les intéressés décrivent comme un don s'inscrit en général dans le cadre de relations sociales durables et dans un cycle d'échanges à long terme. Ainsi, au moment du repiquage du riz dans son bas-fond, tel aîné pourra solliciter l'aide d'un jeune paysan qui lui fera bénéficier de son tour de kune. Souvent ce jeune a été nourri par l'aîné durant une période de sa vie et, en cas de besoin pressant, il pourra encore espérer que l'aîné lui fasse don d'une partie de sa récolte.

\subsection{Le partage des kune au sein des groupes domestiques : comment} « décomposer » les actifs en journée de travail

La kune ne permet pas seulement l'échange de journées de travail entre groupes domestiques mais également le partage de ces journées entre individus qui les composent. Négocié en fonction de l'âge, du sexe et des ressources individuelles, ce partage traduit l'évolution dynamique du groupe.

Une épouse et son mari partagent leur temps de travail sur leurs parcelles respectives. Mais la jeune femme qui vient de rejoindre un groupe domestique comme première épouse se trouve en position défavorable: elle bénéficiera peu de ses propres tours de kune essentiellement consacrés aux parcelles de son mari et de sa belle-mère. De plus, affectée aux tâches domestiques les plus ingrates, elle n'aura guère de temps à consacrer à ses parcelles. Plus tard, quand ses enfants auront grandi, elle cherchera pour son époux de «petites épouses » (yereme huyete) qui la remplaceront dans la kune et assureront à leur tour les tâches domestiques les plus fastidieuses. La première épouse augmentera ainsi progressivement la surface de ses cultures et son autonomie au sein du groupe ${ }^{16}$.

Ce partage dynamique du travail disponible au sein du kuru kuru a également lieu entre les générations. Un jeune homme célibataire, disposant de 5 tours de kune consacrera, sans transiger, les deux premiers aux parcelles du chef du groupe domestique qui en négociera luimême la date et rassemblera les vivres nécessaires à la préparation du repas. La mère - ou la tutrice - du jeune homme, à la tête d'une des marmites du groupe, le sollicitera elle aussi pour le labour de sa parcelle d'arachide. On laissera au jeune son dernier tour de kune pour qu'il

16 Si le rapport de force est défavorable aux jeunes épouses, la négociation reste nécessaire car elles peuvent mettre à exécution la menace d'un retour dans leur hameau d'origine, souvent voisin. En pleine saison des pluies, une telle démission mettrait en péril toutes les entreprises en cours. 
puisse cultiver une parcelle de piment. Les jeunes hommes et les jeunes femmes prennent donc parfois le risque de « voler » des journées de travail aux aîné(e)s du groupe en vendant un tour de kune en cachette. A mesure que les jeunes hommes prennent des responsabilités, qu'ils se marient et élèvent des enfants, leurs aîné(e)s les laisseront jouir d'une part croissante de leur kune. A la tête d'un groupe domestique important, un homme âgé disposera ainsi d'un seul tour de kune de la part de chacun de ses fils, lesquels travailleront avec leur femme, bien souvent sous l'autorité de leur mère, de manière largement autonome. Bien avant l'éclatement du kuru kuru qui suivra le décès de l'aîné se dessine ainsi en pointillés le contour des groupes domestiques en formation.

Naguère circonscrite au sein du kuru kuru sous l'autorité incontestée de l'aîné, la circulation du travail dans les hameaux de la Sella Limba a aujourd'hui pris la forme d'échanges non équivalents de kune entre individus de groupes domestiques, différents ou pas, reflétant l'émergence de stratégies individualistes, contribuant à l'éclatement des groupes domestiques. Mais la marchandisation est limitée et les nombreux échanges de travail restent contraints par les rapports sociaux «traditionnels» intergénérationnels et de genre qui se prolongent au-delà des cycles agricoles.

On observe néanmoins dans certains hameaux de nouvelles formes d'organisation du travail qui indiquent comment la marchandisation des rapports sociaux pourrait s'approfondir dans les années à venir. Certains jeunes hommes préfèrent rejoindre des «kune » de petite taille, 5 personnes par exemple, pour lesquels ils adoptent des règles souples en supprimant le repas ou en remplaçant le riz par un aliment moins coûteux comme le manioc bouilli. Ils se soustraient ainsi à la fois à la nécessité de disposer d'importantes ressources pour mobiliser un tour de kune et au contrôle des aînés qui s'exerce nécessairement dans les grandes kune. Avec le développement de l'orpaillage, on voit même apparaître un nouveau type de rapport social que les paysans appellent le bundali : les travailleurs sont loués à la journée, individuellement, hors de toute kune, et le salaire est négocié à la tâche. Les salaires journaliers du bundali sont calés sur ceux de l'orpaillage et il n'y pas de repas à fournir. L'apparition de tels contrats préfigure peut-être l'aboutissement d'une tendance à la marchandisation complète du travail.

\section{La différenciation économique et sociale des unités de production / consommation aujourd'hui en Sella Limba}

Tandis que l'éclatement des groupes domestiques (kuru kuru) en unités de production et de 
consommation de taille réduite est corrélé à la marchandisation des rapports sociaux, aucun développement significatif des forces productives n'accompagne cette dynamique: la productivité journalière du travail agricole paysan a stagné, voire reculé. L'étude des processus productifs à l'échelle des unités de production et de consommation nous révélera qu'il en résulte une faible différenciation économique et sociale de la paysannerie reposant néanmoins sur une pluralité de mécanismes de captation du travail.

\subsection{Reproduction simple et reproduction élargie: l'économie des processus de} production agricole en Sella Limba

Pour mesurer les résultats économiques des unités de production / consommation, nous distinguerons d'une part la Valeur Ajoutée (VA), c'est-à-dire le résultat du processus technique de production et d'autre part le revenu agricole (RA) qui reflète quant à lui les conditions d'accès aux moyens de production et en conséquence les modalités de l'appropriation sociale du produit (Cochet 2011a et 2012) ${ }^{17}$. De plus, nous distinguerons la part du revenu qui permet la reproduction simple de l'unité d'un cycle agricole à l'autre (c'està-dire sa reproduction à l'identique, dans son fonctionnement social et technique), de celle qui permet d'envisager sa reproduction élargie (le surplus).

Le surplus que peut dégager en moyenne un actif agricole dépend étroitement de la surface qu'il est en mesure de billonner à la houe et de désherber manuellement entre les mois d'août et de mai. Dans les conditions courantes ${ }^{18}$, nous estimons qu'un actif peut cultiver annuellement 0,1 ha en bas-fond et 0,8 ha en terres pluviales pour un produit brut par actif et par an de l'ordre de 460,6€. Après déduction de la valeur des semences $(65,8 €)$, on obtient une valeur ajoutée par actif et par an de 394,7 €, la valeur du capital immobilisé - l'outillage manuel - étant négligeable. La part de la valeur ajoutée qui revient au producteur $(308,7 €)$ est déduite en retranchant la part de la récolte qui est concédée aux aînés du lignage fondateur,

17 La première exprime la création de richesse, c'est-à-dire à la différence entre la valeur de toutes les richesses produites (produit brut) et la valeur des richesses détruites. Le RA correspond à ce qui revient finalement au producteur après le partage de la valeur ajoutée en fonction des rapports sociaux qui déterminent l'accès aux moyens de production (force de travail extérieure à l'unité de production, accès au foncier, éventuellement accès au capital).

18 Tous les résultats qui suivent proviennent de l'analyse des 58 études de cas menés en Sella Limba. L'exposition complète de la méthode et des hypothèses nécessaires pour les calculs économiques excèdent les limites de cet article (Cochet, 2011a et 2012). Signalons néanmoins qu'il repose, pour chaque unité de production / consommation, sur un inventaire précis des actifs et des consommateurs en présence, des parcelles cultivées par chaque individu et de l'origine et de la destination de la force de travail mobilisée. VA et RA correspondent à la valorisation, le plus souvent au prix du marché de détail, de toutes les productions : cultures en rotation et en association, récoltes vendues, intra ou auto-consommées, données, ... 
distributeurs des droits d'usufruit de la terre, ainsi que les divers dons consentis au moment de la récolte. La quasi-totalité de ce RA $(92 \%)$ est consacrée à la subsistance des membres du groupe (producteurs, pré- et post-producteurs) ${ }^{19}$. Le nombre moyen de consommateurs ${ }^{20}$ par actif est, aujourd'hui en Sella Limba, d'environ de 1,9 (soit 0,9 inactif /actif). Ce rapport varie sensiblement d'une unité à l'autre et nous reviendrons sur ce point essentiel. Dans les conditions (techniques, économiques et sociales) actuelles, le surplus moyen par actif agricole est donc très faible (de l'ordre de $24,7 €$ ) et la reproduction simple des unités est à peine assurée (figure 2).

Cette situation moyenne ignore les effets des nombreux aléas (climatique, économique, politique, etc.) et de la morbidité (maladie, accidents) qui pèsent sur la production et les travailleurs. Étant donné la faiblesse du surplus dégagé, le moindre accident peut menacer à court terme la survie de l'unité de production / consommation. Dans ces conditions, les différentes formes de solidarité qui se tissent au sein de la paysannerie restent une nécessité. Les dons concédés à la récolte, dont on a vu l'importance dans la valeur ajoutée totale, constituent la forme la plus visible de cette solidarité. Ainsi, la précarité des unités explique, comme on va le voir, la coexistence de stratégies en apparence contradictoires.

19 Soit, au prix du marché, $126 \mathrm{~kg}$ de riz blanc, $14 \mathrm{~kg}$ d'huile de palme, $18 \mathrm{~kg}$ d'arachide coque, $90 \mathrm{~kg}$ de manioc frais, $6 \mathrm{~kg}$ de pois cajan et l'équivalent de 41,3€ d'ingrédients achetés (poissons, glutamate, sel, ...). Les autres besoins vitaux, comme l'habillement ou les produits d'hygiène (hors soins médicaux), ont un coût négligeable. 20 Pondéré par l'âge des consommateurs ( 0,5 pour les enfants qui ne participent pas encore au kune, 1 pour tous les autres). Cette pondération un peu grossière est inspirée de la manière dont les cuisinières calculent la ration en riz pour leur marmite (en cup et demi cup de riz décortiqué). 


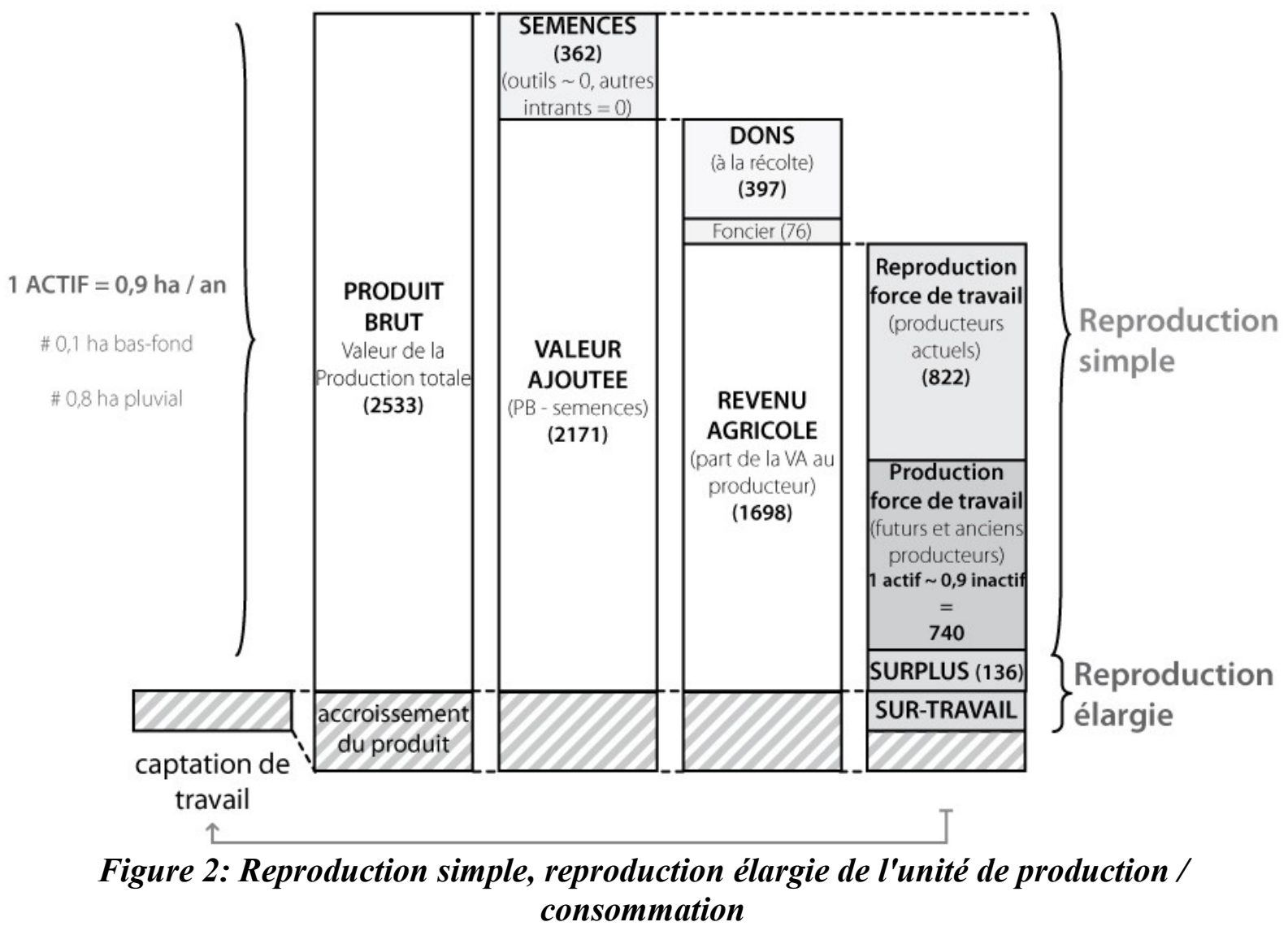

Ce raisonnement correspond à un producteur ou à un groupe de producteur qui emploierait sa seul force de travail sur ses seules parcelles. Or, comme on l'a vu, avec l'éclatement des kuru kuru de ces dernières décennies, les échanges de journée de travail par le biais des kune se sont développés. Afin d'augmenter le surplus qu'ils dégagent, les paysans tentent de consacrer une part de leur récolte à la captation de travail extérieur, entre mai et août, quand le coût d'opportunité de la journée de travail est maximum.

A chaque cycle de culture, les ressources injectées dans le processus de production pour capter du travail proviennent du surplus dégagé durant le cycle de culture précédent. Il y a donc potentiellement accumulation de capital circulant (vivrier et argent), c'est-à-dire reproduction élargie de l'unité de production / consommation. En absence d'accès à des moyens de production plus performants, cette accumulation est la seule source potentielle de différenciation au sein de la paysannerie. Pour estimer l'ampleur de cette accumulation différentielle, et comprendre sous qu'elle forme elle se cristallise, il faut d'abord mesurer la place relative des ressources consacrées à la captation de travail, le surplus ainsi créé, et analyser son utilisation. 
5.2 La faiblesse du surplus limite l'impact de la circulation de la force de travail sur le $\underline{\text { Revenu Agricole }}$

Le travail échangé entre les unités de production circule via les kune et le coût de la force de travail correspond soit à l'achat direct de tours de kune, soit à la préparation d'un repas. En 2011, une kune valait $0,9 €$ / travailleur et par jour et la préparation du repas de mi-journée 0,4 $€$ / travailleur. Les unités de production les mieux dotées en capital circulant (riz ou trésorerie) consacraient jusqu'à $272,7 €$ à la captation de travail, soit une centaine de journées de travail achetées et plus de 200 repas préparés entre mai et août. A l'opposé, d'autres paysans ne disposaient pas des ressources pour la préparation du repas de la kune et devaient parfois vendre un ou plusieurs tours de kune, certains obtenant de cette vente jusqu'à environ $55 €$ (correspondant à plus de 70 jours de travail payés en monétaire, en semences ou en vivrier).

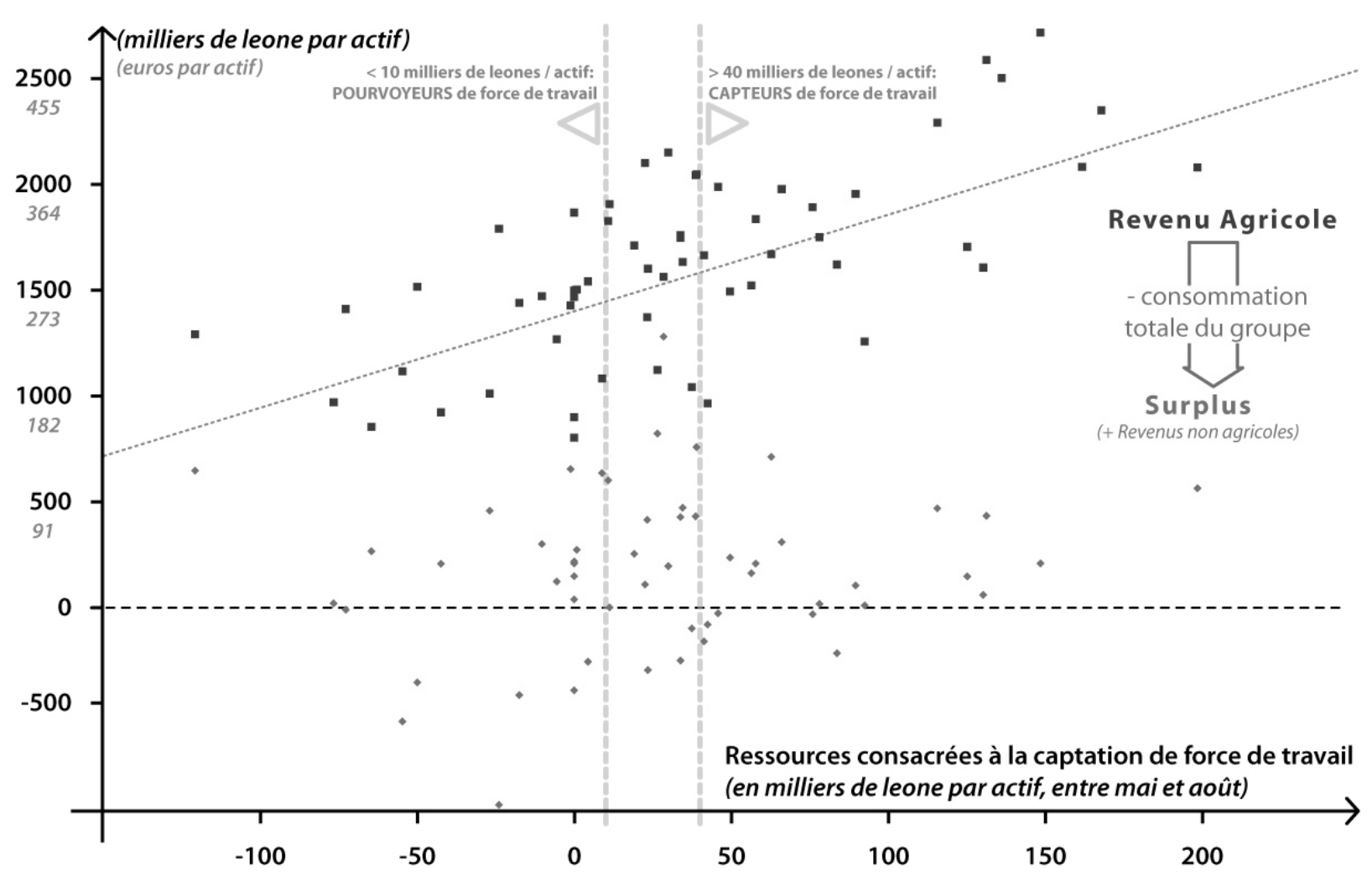

Figure 3: Revenu Agricole et Surplus par actif domestique en fonction des ressources consacrées par actif domestique pour la captation de force de travail entre mai et août

La figure 3 indique que le RA/actif d'une unité de production augmente régulièrement 
avec la quantité de travail extérieur capté ${ }^{21}$. L'essentiel de ce RA est utilisé, comme on l'a vu, à l'alimentation (autoconsommation et achat de vivrier). Si l'on retranche au RA total de l'unité de production/consommation la somme minimum nécessaire à la subsistance de tous ses membres ${ }^{22}$ et qu'on ajoute les revenus non-agricoles ${ }^{23}$, on obtient le « surplus ».

La figure 3 indique la faiblesse générale du niveau des surplus et sa non-corrélation d'avec le niveau de captation de travail. Quelques unités, à la fois bien dotées en ressources et ayant peu de personnes à charge par actif, dégagent un surplus significatif (environ $135 €$ / actif et par an) mais plus nombreuses sont celles dont le surplus est faible (inférieur à $40 €$ ), voire négatif. Pour ces dernières, la solidarité des réseaux de parenté et de voisinage peut aider à passer une année exceptionnellement difficile. Mais lorsque le hiatus entre capacité productive et nombre de bouches à nourrir devient structurel, les unités de production/consommation éclatent : divorce des épouses qui rejoignent avec leur progéniture un autre foyer, placement d'enfants chez un oncle plus prospère ou encore par la fuite des jeunes actifs vers les mines ou vers Freetown.

Parmi les unités qui dégagent un surplus, la moyenne se situe autour de $60 €$ / actif et par an. Il faut comparer ce chiffre à la valeur des biens matériels auxquels les paysans aspirent aujourd'hui dans les campagnes sierra-léonaise. Un homme mûr doit posséder une maison « moderne », couverte de tôles dont le coût moyen s'élève à 363,6€. Pour acquérir ce bien, la plupart des paysans forment des tontines (hosusu) et épargnent 5 à 10 ans. Les bonnes années, une minorité de paysans pourra également se procurer une chèvre (entre 18 et $36 €$ ). Une moto $125 \mathrm{cc}$, moyen de transport précieux pour aller chercher les meilleurs prix sur les marchés régionaux, vaut plus de $900 €$ et reste hors de portée des paysans.

Même pour la catégorie des paysans la mieux dotée en ressources productives, le niveau des surplus interdit l'accès à des moyens de production plus performants et ne permet pas d'envisager un processus de reproduction élargie significatif. Non seulement le nombre de journées captées reste marginal au regard du travail fourni par les actifs domestiques mais encore la productivité journalière du travail restant très faible, le surproduit issu de la captation de journées de travail ne représente qu'une faible part du revenu des groupes

21 Évidemment, pour un même niveau de dépense dans la captation de force de travail on observe des variations liées à de légères différences d'assolements, d'accès à des prix de vente différents ou encore d'équilibre entre hommes et femmes au sein des unités.

22 Il s'agit ici du nombre réel et non plus un nombre moyen de consommateurs de chaque unité.

23 Les revenus de l'orpaillage ont été estimés en considérant qu'un jeune actif travaillait 100 jours dans les mines tout en continuant d'exploiter ses parcelles. 
domestiques les mieux placés. La marchandisation des rapports sociaux ne correspond donc pas à l'émergence, au sein de la paysannerie, d'une classe d'employeurs qui ferait face à une classe de paysans en voie de prolétarisation mais plutôt à une déstructuration générale où les unités les mieux dotées assurent, bon an mal an, leur reproduction simple tandis que la plupart des autres survivent, vaille que vaille, dans des conditions toujours plus dégradées.

\subsection{Captation ponctuelle de force de travail ou de d'énergie de travail en formation :} des stratégies d'accumulation hybrides

Outre la faiblesse des surplus que dégagent les unités de production/consommation, la figure 3 indique également que, contrairement au RA total, le surplus n'est pas corrélé à la quantité des ressources engagées dans la captation de travail à l'extérieur du cercle domestique. Les unités de production qui captent de la force de travail ne dégagent pas un surplus supérieur à celles qui en cèdent. Cette situation s'explique par des différences dans le nombre de bouches à nourrir par actif. En moyenne, le rapport consommateurs / actif est de 1,9. Mais dans certaines unités ce rapport chute à moins de 1,5 (par exemple un couple avec 2 enfants) tandis que dans d'autres il grimpe au-delà de 2,5 (un couple avec 6 enfants). Ces disparités ne sont pas le produit d'une différence de croît démographique endogène aux unités de production/consommation ${ }^{24}$. Elles résultent de réarrangements permanents au travers, notamment, de la circulation des enfants entre unités. Comme dans un grand nombre de sociétés paysannes ouest-africaines, les rejetons ne sont pas élevés systématiquement par leurs géniteurs ou par eux pas seulement. Une enquête que nous avons menée auprès de 148 femmes adultes révèle que sur leurs 223 rejetons vivants encore à charge d'un adulte (enfants et adolescents célibataires), 61 d'entre eux (27\%) sont élevés dans une autre unité que celle de leur génitrice. A l'opposé, ces 148 femmes adultes élèvent 72 enfants $(30 \%$ des enfants à leur charge) nés hors de leurs unités respectives ${ }^{25}$. Comme ailleurs en Sierra Leone (Bledsoe \& Isiugo-Abanihe 1989), les femmes âgées, notamment les grands-mères maternelles, sont les bénéficiaires fréquentes de cette circulation. Souvent les enfants sont élevés par plusieurs femmes durant toute leur vie pré-productive et nourrir au quotidien un enfant n'est pas la seule manière de contribuer à son élevage : on peut également fournir l'argent et le riz nécessaire à

24 Comme c'est le cas, par exemple, dans le modèle de la famille paysanne de Tchayanov (1990).

25 C'est une estimation basse du phénomène qui correspond aux cas de changement de résidence de l'enfant. Entre les marmites d'une même unité de résidence, la circulation des enfants est plus discrète et encore plus réversible. Ces chiffres sont par ailleurs cohérents avec les résultats d'autres enquêtes réalisées en Sierra Leone. Chez les mende de l'est du pays, entre 30 et $45 \%$ d'enfants sont « fosterés »(Bledsoe \& Isiugo-Abanihe 1989, Isaac \& Conrad 1982). 
son initiation ou encore participer aux frais de sa scolarisation.

Sans exclure la dimension affective, les grands-mères concernées décrivent explicitement l'énergie et les ressources qu'elles consacrent à élever ces enfants comme un investissement. Quand elles se plaignent d'enfants qui ne donnent pas « de nouvelles » depuis leur départ à Freetown, elles leur reprochent de ne recevoir ni argent, ni riz, ni vêtements. De la même manière, elles disent d'un enfant décédé avant d'avoir pu travailler ou d'un adolescent qui a fui sans «dire au-revoir », qu'il est "gâté », comme pour une récolte ou n'importe quel autre investissement perdu.

Dans la mesure où une partie du RA sert, non pas à la captation immédiate de force de travail mais à l'élevage des pré-productifs, c'est-à-dire à la formation d'énergie de travail dont la jouissance est reportée, donc incertaine, mais potentiellement inscrite sur plusieurs années, voire sur plusieurs générations, on peut parler de stratégies d'accumulation hybrides : productive et viagère. Ces deux stratégies, captation de journées de travail d'une part, adoption de futurs actifs d'autre part, qui se développent à des pas de temps différents, sont en partie contradictoires : la part du RA «mangée» par les enfants non productifs est indisponible pour la captation immédiate de journées de travail. En partie seulement car c'est bien la captation de travail et l'appropriation d'un surproduit qui autorisent la captation de cette énergie de travail en devenir et la consolidation de l'unité de production/consommation

\subsection{La permanence actuelle des anciens rapports sociaux dans les grands groupes} domestiques

Dans leurs discours, les aînés continuent d'accorder beaucoup d'importance à « l'unité de la maison » et ils désapprouvent l'ambition des jeunes gens à la recherche d'une émancipation toujours prématurée à leurs yeux. Il y a donc un paradoxe : si la captation ponctuelle de force de travail permet aujourd'hui à quelques paysans d'accumuler à court terme du capital circulant, à long terme, réunir un grand nombre d'individus sous leur autorité continue d'être synonyme de prospérité.

L'éclatement des kuru kuru a globalement donné naissance à des unités de production/consommation plus petites, mais la situation est hétérogène. La majorité des unités rassemblent de 2 à 4 actifs, généralement organisées autour d'un couple monogame auquel s'ajoute un frère ou un enfant en âge de travailler. Quelques hommes, âgés et polygames, rassemblent sous leur autorité de 5 à 10 actifs. Ces dernières unités semblent reproduire les 
rapports sociaux qui prévalaient au sein des anciens kuru kuru: tous les actifs contribuent au travail sur les parcelles du doyen qui redistribue le produit vivrier via les femmes âgées du groupe qui chacune contrôle une marmite. Cependant, aussi bien pour leur subsistance que pour leurs revenus monétaires, les marmites sont aujourd'hui plus autonomes que par le passé. Les disparités économiques entre marmites d'une même unité peuvent d'ailleurs être aussi importantes qu'entre unités elles-mêmes. Mieux, chaque travailleur dispose individuellement de ses propres parcelles dont la taille varie en fonction de son statut et de ses ressources propres. Nous avons vu comment l'institution de la kune permet la circulation de la force de travail disponible au sein de ces unités complexes.

Si individus et sous-unités jouissent d'une si grande autonomie, comment expliquer la persistance des kuru kuru contemporains? Les figures 5 et 6 montrent que, proportionnellement au nombre d'actifs, le nombre d'enfants à charge comme le niveau des ressources investies pour capter du travail sont plus importants pour ces unités. Aucune d'entre elles n'est pourvoyeuse nette d'enfants ou de force de travail. Elles sont au contraire les principales destinataires à la fois des enfants et des journées de travail en circulation.

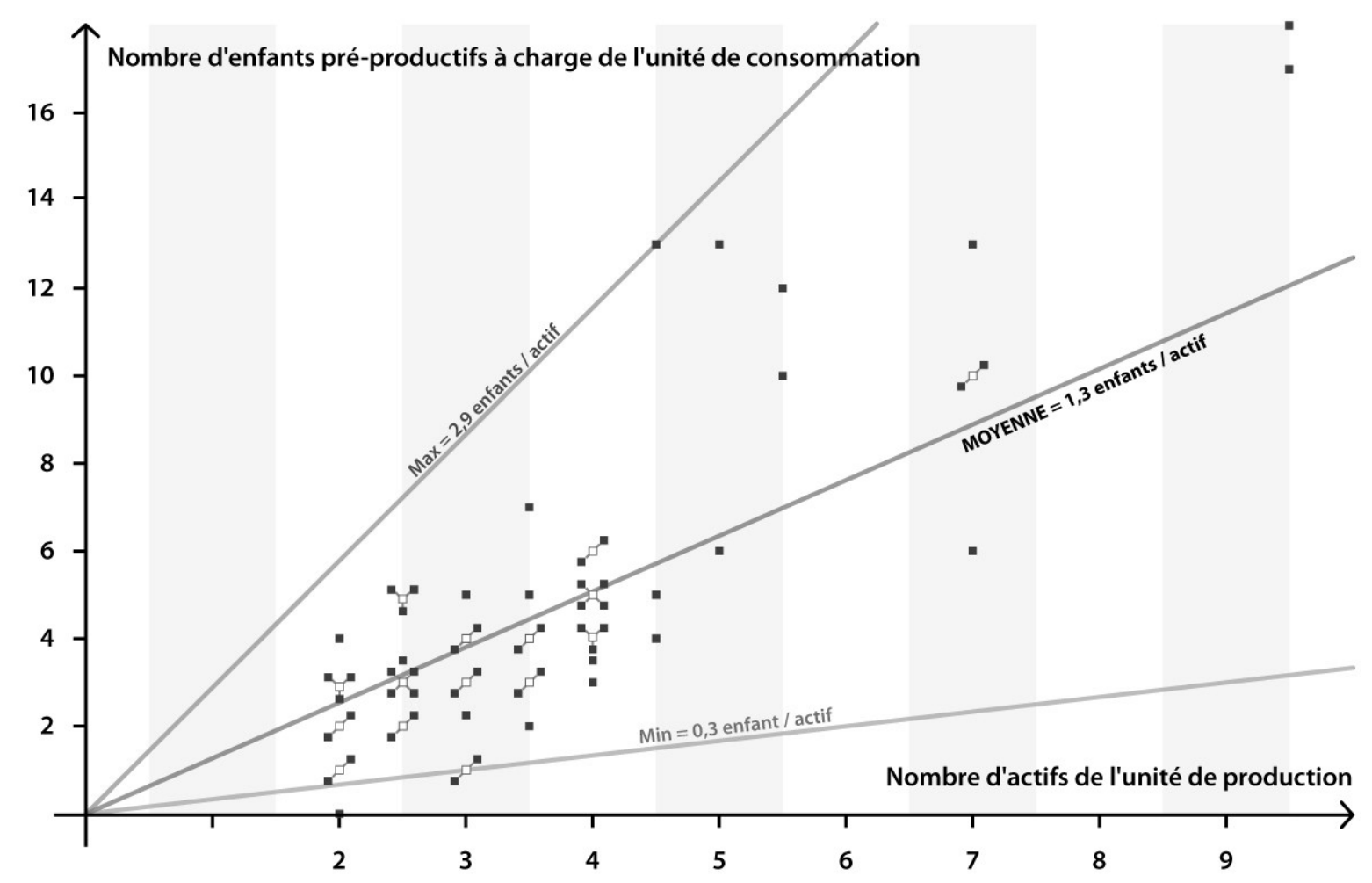

Figure 4: Nombre d'enfants pré-productifs à charge en fonction du nombre d'actifs (par unité de production / consommation) 


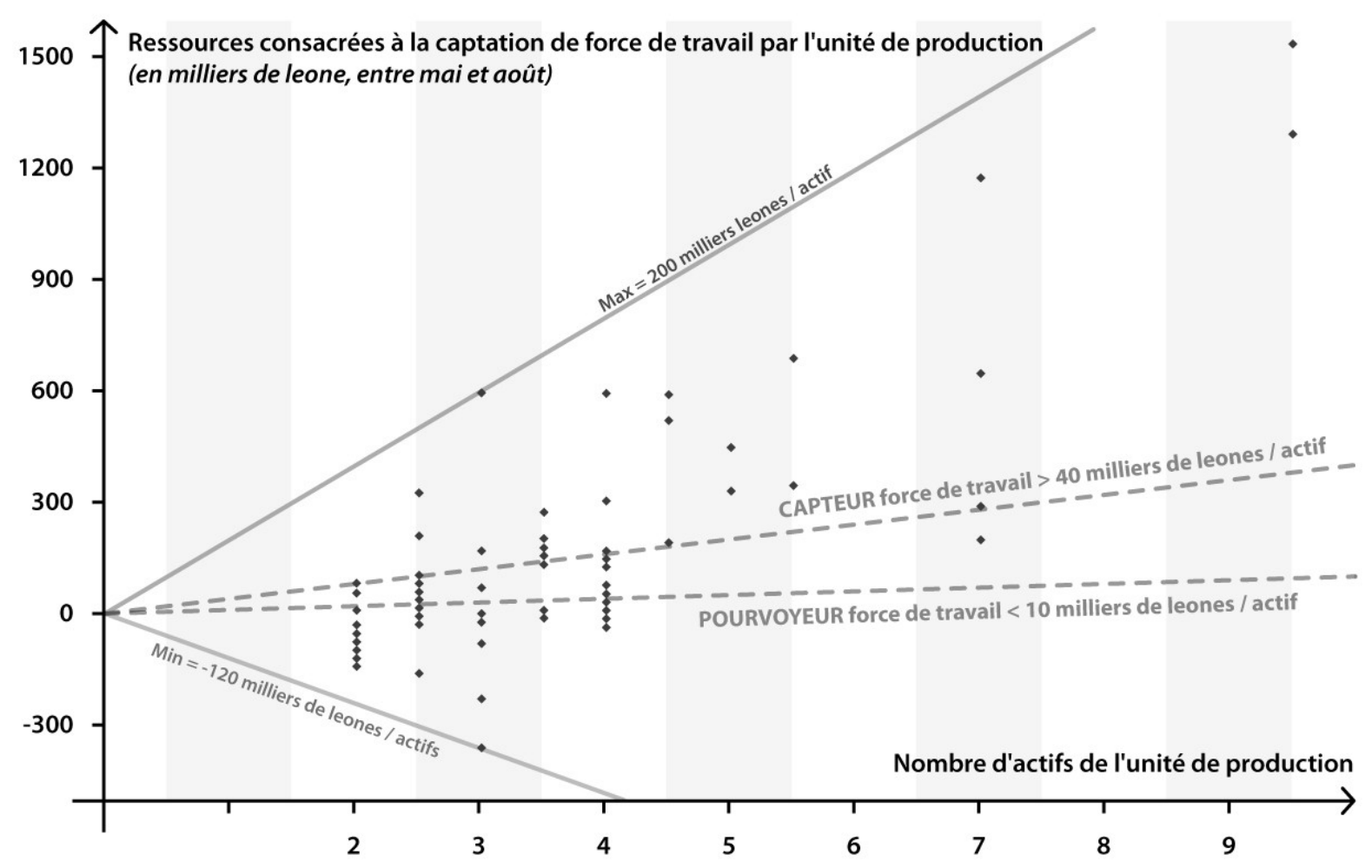

Figure 5: Ressources consacrées à la captation de force de travail entre ami et août en fonction du nombre d'actifs (par unité de production / consommation)

Cette situation s'explique par une économie d'échelle. La force de travail se présente en quantités discrètes : il est en effet encore impossible d'acheter une heure ou une journée de travail. Il faut mobiliser d'un coup les ressources nécessaires à l'achat un tour de kune dans son intégralité (18,2 € par exemple pour un tour de kune de 20 membres). Ainsi, en raison de la faiblesse des surplus individuels et du caractère discret de la force de travail, seules les unités de production/consommation qui «mutualisent» le surplus d'un grand nombre d'actifs ont la capacité de capter du travail. Les petites unités, même si elles dégagent un surplus, n'ont pas cette capacité. Ce raisonnement vaut également pour l'adoption d'un enfant.

La mutualisation repose sur la capacité des aînés à réunir sous leur autorité, en activant les rapports sociaux d'antériorité, même déformés et manipulés, un nombre suffisant d'actifs. Mais ces pseudo-kuru kuru contemporains sont soumis à de très fortes tensions : les femmes veillent à ce que leur propre progéniture ne soit pas lésée et les jeunes travailleurs sont souvent tentés d'abandonner les aînés à leurs parcelles pour émigrer à Freetown. Ainsi l'«unité » domestique, tant loué par les hommes âgés, dépend largement de l'intérêt que trouvent les femmes et les cadets à rester sous leur autorité et donc des récoltes qu'ils 
obtiennent chaque année. Les vieux sella limba le répètent : s’il n’y a pas de riz, il n'y a pas d'unité.

A la mort de l'aîné, ces grandes unités éclatent en unités plus petites de 2 à 4 actifs. Les positions respectives des individus dans l'unité d'origine déterminent alors des trajectoires divergentes et la diversité des situations dérivées (représentées sur la gauche des figures 4 et 5). Les fils aînés, souvent issus des premières épouses du doyen décédé, bénéficient de la force de travail des cadets de leur phratrie élevés par les hommes et les femmes de la génération précédente, surtout leur mère. Durant plusieurs années, les jeunes unités de production qu'ils dirigent, au ratio inactifs/actifs faible, peuvent enclencher le processus de captation de force de travail et d'accumulation. On retrouve ici les unités de production qui dégagent les surplus par actif domestique les plus élevés. A l'inverse, les cadets, notamment ceux nés des dernières épouses du doyen, ne peuvent pas suivre pareille trajectoire. Ils ont le choix entre travailler sous l'autorité d'un ainé ou constituer de petites unités caractérisées par un déficit de capital circulant. C'est parmi ces unités-là que se trouvent les pourvoyeurs de force de travail et d'enfants pré-productifs, dont elle assume difficilement l'élevage.

Avec l'éclatement prochain des dernières unités de production fonctionnant peu ou prou sur le modèle des kuru kuru de naguère, assistera-t-on à l'amorce d'une polarisation durable de la paysannerie? Si notre analyse est juste, ces processus se déroulant sans progrès de la productivité du travail et dans des conditions d'intégration au marché toujours aussi défavorables, les jeunes paysans les plus prospères ne pourront durablement poursuivre une stratégie productive et individualiste à court terme. Il probable qu'ils reproduiront le schéma d'accumulation d'énergie humaine à long terme de la génération précédente en adoptant des pré-productifs.

\section{Conclusion}

Dans les agricultures manuelles africaines caractérisées par une très faible productivité du travail, comme au nord de la Sierra Leone, les stratégies socio-économiques des producteurs sont complexes. La situation de certaines unités de production/consommation leur permet de développer des stratégies d'accumulation, certes limitée, de capital productif à court terme. Parallèlement, elles continuent de mettre en place des stratégies à long terme de captation d'individus pré-productifs. Ces deux types de stratégies, à la fois complémentaires et concurrentielles, mobilisent une combinaison évolutive de rapports sociaux «traditionnels » 
et «modernes ». D'autres unités se trouvent dans l'incapacité de développer l'une ou l'autre de ces stratégies et luttent pour leur simple survie.

La marchandisation des rapports sociaux, quelquefois chaotique, semble devoir s'accentuer. En témoignent les effets du développement récent de l'orpaillage local. La vente de journée de travail dans les mines d'or fait désormais partie de la vie d'un grand nombre de jeunes hommes. Bien qu'encore marginales, ces nouvelles modalités de circulation de travail, purement salariales, pénètrent la production agricole paysanne. Dans les prochaines années, l'intégration économique des paysanneries sierra léonaises pourrait connaître une nouvelle phase d'accélération avec de grands projets agro-industriels et la contractualisation de l'agriculture que cherche à développer le gouvernement sierra-léonais (Baxter 2011, MAFFS 2009 : 34). Ces paysanneries, marginalisées et aujourd'hui menacées d'être dépossédées de leur terre, continueront sans doute à jouer le rôle de fournisseurs de denrées et force de travail bon marché pour le capital des mines ou de l'agro-business. Dans ce cadre, l'émergence au sein de la paysannerie d'une classe de producteurs prospères et salariant eux-mêmes leurs voisins démunis n'est guère probable. L'involution de la paysannerie sella limba devrait donc se prolonger par un exode, une paupérisation et une prolétarisation croissantes et rejoindre de nombreuses paysannerie du Sud dans un processus évoquant plus les enclosures qu'une quelconque « koulakisation » (Kenney-Lazar 2011).

\section{Références bibliographique}

ALIE, J.A.D., 1990, A new history of Sierra Leone, Londres, Macmillan.

BAXTER J., 2011, Understanding Land Investment Deals in Africa. Country Report, Sierra Leone, Oakland, The Oakland Institute.

BernsteIn, H., 2010, Class dynamics of agrarian change, Sterling, Kumarian Press.

BINDER, K., 1989, The vicious cricle of hunger and indebtedness. Analysis of the farming system of South-East Bombali District. Sierra Leone, Wageningen, ICRA.

Bledsoe, C.H. \& IsiugO-ABAniHe, U., 1989, Strategies of Child Fosterage among mende Grannies in Sierra Leone, in: LeSthAEGHE, R.J. (dir.), Reproduction and Social Organization in sub-Saharan Africa, Berkeley, University of California Press : pp.442-474.

COCHet H., 2011a, L'agriculture comparée, Versailles, Quae.

COCHET H., 2012, The 'systeme agraire' concept in francophone peasant studies, Geoforum 43 : pp.128-136. 
DELARUE J., 2007, Mise au point d'une méthode d'évaluation systémique d'impact des projets de développement agricole sur le revenu des producteurs: étude de cas en région kpèlè (République de Guinée), Paris, AgroParisTech.

DeVEnEAUX, G.K., 1973, The political and social impact of the colony in the Northern Sierra Leone 1821-1896, Ann Arbor, University Microfilm.

ISAAC, B.L. \& CONRAD, S.R., 1982, Child fosterage among the Mende of Upper Bambara chiefdom, Sierra Leone: rural-urban and occupational comparisons, Ethnology 21 (3) : pp.243-257.

KenNeY-LAZAR, M., 2011, Dispossession, semi-proletarianization and enclosure: private accumulation and the land grab in Laos, présenté à l'International Conference on Global Land Grabbing, LDPI / University of Sussex, 6-8 avril 2011, www.future-agricultures.org/papersand-presentations (page visité le 1/7/2013).

LEACH, M., 1994, Rainforest Relations: Gender and Resource Use Among the Mende of Gola, Sierra Leone, Edinburgh, University Press.

LEVI, J., 1975, African agriculture misunderstood: policy in Sierra Leone, Stanford, Food Research Institute, Stanford University.

MAFFS, 2009, National Sustainable Agriculture Development Plan 2010-2030.Sierra

Leone's Comprehensive African Agriculture Development Programme, Freetown, MAFFS.

MAZOYER, M., ROUDART, L., 2002, Histoire des agricultures du monde: du néolithique à la crise contemporaine, Paris, Seuil.

McCulloch M., 1964, The Peoples of Sierra Leone Protectorate, Londres, International African Institute/

MeILlassoux, C., 1975, Femmes, greniers et capitaux, Paris, Maspero.

Palliere, A., 2013, Au-delà de Malthus et Boserup: comprendre les dynamiques agroécologiques d'un territoire. Un cas d'étude en Sella Limba, Sierra Leone, présenté à la rencontre nationale des Jeunes Chercheurs en Études Africaines, 11-12 janvier 2013, Paris, jcea2013.sciencesconf.org/conference/jcea2013/pages/augustin_palliere_VF_.pdf (page consulté le 22 juin 2013)

Paul, J.-L, 2003, Anthropologie historique des Hautes Terres de Tanzanie orientale: Stratégies de peuplement et reproduction sociale chez les Luguru matrilinéaires, Paris, Karthala.

Pillot D., 1993, Paysans, Systèmes et Crise - Travaux sur l'agraire haïtien, Tome 2, Stragégies et logiques sociales, Pointe-à-Pitre/Port-au-Prince, SACAD/FAMV. 
RICHARDS, P., 1996, Fighting for the rain forest, Oxford, James Currey.

RIDDELl, J.B., 1970, The spatial dynamics of modernization in Sierra Leone: structure, diffusion, and response, Evanston, Northwestern University Press.

SÉBiLlotTE, M., 1977, Jachère, système de culture, système de production, Journal d'Agriculture Tropicale et de Botanique Appliquée (JATBA), 24 (2-3) : pp.241-264.

SiBANDA, M.J.M., 1979, Dependency and Underdevelopment in Northwestern Sierra Leone, 1896-1939, African Affairs 78 : pp.481-492.

TChAyANOV, A.V., 1990 [1925], L'organisation de l'économie paysanne, Paris, Éditions de la Maison des sciences de l'homme.

ZaCK-Williams, A.B., 1982, Merchant Capital and Underdevelopment in Sierra Leone, Review of African Political Economy 25 : pp.74-82. 